\title{
Merging Massive Star Clusters as Building Blocks of Dwarf Galaxies?
}

\author{
M. Fellhauer and P. Kroupa \\ Inst. Theor. Phys. \& Astrophys., Univ. Kiel, Germany
}

November 30, 2018

\begin{abstract}
Recent spectroscopic observations of galaxies in the Fornax-Cluster reveal nearly unresolved 'star-like' objects with red-shifts appropriate to the FornaxCluster. These objects have intrinsic sizes of $\approx 100 \mathrm{pc}$ and absolute B-band magnitudes in the range $-14<\mathrm{M}_{\mathrm{B}}<-11.5 \mathrm{mag}$ and lower limits for the central surface brightness $\mu_{\mathrm{B}} \geq 23 \mathrm{mag} / \operatorname{arcsec}^{2}$ (Phillipps et al. 2001, Hilker et al. 1999), and so appear to constitute a new population of ultra-compact dwarf galaxies (UCDs). Such compact dwarfs were predicted to form from the amalgamation of stellar super-clusters (= clusters of star clusters; not to confuse with super stellar clusters (SSC)) by P. Kroupa (1998), which are rich aggregates of young massive star clusters (YMCs) that can form in collisions between gas-rich galaxies. Here we present the evolution of super-clusters in a tidal field. The YMCs merge on a few super-cluster crossing times. Super-clusters that are initially as concentrated and massive as Knot S in the interacting Antennae galaxies (Whitmore et al. 1999) evolve to merger objects that are long-lived and show properties comparable to the newly discovered UCDs.
\end{abstract}

Keywords: Galaxies: evolution - Galaxies: formation - Galaxies: Interaction Galaxies: Star Clusters - Methods: N-body simulations

\section{Introduction}

Spectroscopic surveys of the Fornax-Cluster revealed 5 compact objects which have spectra typical for late-type metal-rich and old stellar populations, are marginally resolved and have red-shifts comparable to the Fornax-Cluster, ruling out either faint background galaxies or foreground stars. Four of these may be globular clusters at the very bright end of the luminosity function (Mieske et al. 2001) but one is resolved with a diameter of $\approx 300$ pc. Phillipps et al. stated that these objects form a new class of objects, namely Ultra-Compact Dwarf Galaxies (UCD). The UCDs have intrinsic sizes of around $100 \mathrm{pc}$, absolute B-band magnitudes of $-14<\mathrm{M}_{\mathrm{B}}<-11.5 \mathrm{mag}$ and lower limits (due to failure to resolve their cores) of the central surface-brightness $\mu_{\mathrm{B}} \geq 23 \mathrm{mag} / \operatorname{arcsec}^{2}$. Further analysis of photographic plates show no sign of low-luminosity envelopes around these objects which rules out the possibility that they are nucleated dwarf ellipticals $(\mathrm{dE}, \mathrm{N})$ with faint envelopes. Therefore, these objects are either extremely compact 
dwarf galaxies or extremely large and massive $\left(10^{7}-10^{8} M_{\odot}\right.$, assuming $\left.M / L_{\mathrm{B}}=3\right)$ star clusters. In the $\mu_{\mathrm{B}}-M_{\mathrm{B}}$ diagram they fall in the empty region between 'ordinary' dwarf galaxies and globular clusters.

High-resolution HST-images of the star forming regions in interacting galaxies like the Antennae (Whitmore et al. 1999; Zhang \& Fall 1999) or Stephan's Quintet (Gallagher et al. 2001) resolve some of these regions into dozens to hundreds of young massive star clusters. According to Whitmore et al. the individual clusters have effective radii of about $4 \mathrm{pc}$ and masses $10^{4}-10^{6} \mathrm{M}_{\odot}$, with a mass-spectrum following a steep power law $\Psi_{M} \propto M^{-2}$ (Zhang \& Fall 1999). The striking point is that these young star clusters are themselves clustered into groups of a few to several hundred star clusters spanning projected regions of a few $100 \mathrm{pc}$, with a higher cluster concentration at the centre. Measurements of the relative velocities between the star clusters within such objects are now becoming available. Preliminary results indicate $\approx 20 \mathrm{~km} / \mathrm{s}$ (B. Whitmore, private communication), which is consistent with virial masses $\approx 10^{7} M_{\odot}$. Age determinations in the Antennae show that these star clusters are extremely young (3-7 Myr). While in the Antennae young massive star clusters are preferably found in the central region of the interacting pair, NGC 7319 in Stephan's Quintet has young star clusters in the long tidal arm and the intra-group region north of NGC 7319 (Gallagher et al. 2001).

\section{Setup}

The orbital integration of the particles is performed with the particlemesh code Superbox (Fellhauer et al. 2000). In Superbox densities are derived on Cartesian grids using the nearest-grid-point (NGP) scheme. From these density arrays the potential is calculated via the fast Fourier-transformation. Forces are obtained using higher-order differentiation based on the NGP scheme but comparable in precision with standard CIC (cloud-in-cell) algorithms. The particles are integrated using a fixed time-step Leap-Frog algorithm. For an improved resolution at the regions of interest, a hierarchical grid-architecture with two levels of high resolution sub-grids are used for each star cluster in the supercluster. These sub-grids track the density maxima of the individual star clusters, and are adjusted at the beginning of the computation to meet individual requirements.

The young massive star clusters are modeled as Plummer-spheres with Plummer-radii of $4 \mathrm{pc}$ and cutoff-radii of $20 \mathrm{pc}$. They have masses of $10^{6} \mathrm{M}_{\odot}$ and $3.2 \cdot 10^{5} \mathrm{M}_{\odot}$ to mimic a mass spectrum. 32 star clusters (29 light and 3 heavy) are placed together in a sphere with places and 
relative velocities according to a Plummer-distribution with a Plummerradius of $50 \mathrm{pc}$ and a cutoff-radius of $250 \mathrm{pc}$. This configuration is than placed on an highly eccentric orbit around a parent galaxy which is modeled as a rigid spherical potential. The model starts at an apogalacticon position at $20 \mathrm{kpc}$ and passes perigalacticon at $2 \mathrm{kpc}$ distance. The total integration time was 10 Gyr.

\section{The merger object}

Within 500 Myr all 32 clusters have merged, building up a dense, compact merger object. The total mass of the merger object is $\approx 10^{7} \mathrm{M}_{\odot}$ after formation. But the object looses mass with every perigalacticon passage. Time-averaged the best fit is a linear decrease of the mass. After 10 Gyr the object has lost about half of its mass but it still forms a stable and bound object. The surface density after 10 Gyr can be wellfitted with a King profile with a tidal radius of about 400 pc and a core radius of about $11 \mathrm{pc}$. There is a deviation from a simple King profile in the range between the tidal radius at perigalacticon $(\approx 150 \mathrm{pc})$ and the actual tidal radius but this is due to the fact that a simple King profile does not account for a varying tidal field. Fitting the different parts of the profile shows that an exponential with a central surface density of about $4500 \mathrm{M}_{\odot} / \mathrm{pc}^{2}$ and an exponential scale length of $12.5 \mathrm{pc}$ fits the very inner part best. Out to the tidal radius of the last perigalacticon a profile following a $r^{-2}$ power law is the best choice of fitting. The outermost part is best fitted by a steep power-law proportional to $r^{-4.5}$ out to the actual tidal radius. Beyond that the surface density turns over to a flat distribution of underlying extra-tidal stars. This can be understood by this material gets unbound near perigalacticon. But the stars do not leave this region immediately and some are recaptured, when the object is near apogalacticon. The central space density of this object is about $250 \mathrm{M}_{\odot} / \mathrm{pc}^{3}$. We also analyzed the line-of-sight velocity dispersion distribution. The central velocity dispersion is about $12 \mathrm{~km} / \mathrm{s}$ and follows an exponential profile but with an exponential scale length of almost the size of the system. Measuring the line-of-sight velocity dispersion reveals unbound stars even within the merger object. This leads to an artificial rise of the velocity dispersion in the outer parts (outside the core). Even if stars with a clear deviation from the velocity distribution of the bound stars are removed, a rising velocity dispersion near the tidal radius remains. 


\section{Conclusions \& Outlook}

Our models show that star cluster aggregates like the ones found in the Antennae or Stephan's Quintet are very likely to merge thereby building up merger objects which have sizes spanning from massive globular clusters (like Omega Centaurus) to ultra-compact dwarf galaxies (as found in the Fornax Cluster) up to very small dwarf ellipticals. This shows that interactions between gas-rich galaxies followed by interaction-triggered star-formation are possible origins for secondgeneration dwarf galaxies. The main difference between dwarf galaxies generated by such events and primordial dwarfs is that they are not dark matter dominated. In the young universe the interaction rate between galaxies must have been higher, with ubignitous formation of second-generation dwarf galaxies. Our simulations show that even the smaller ones are able to survive in a strong and varying tidal field for more than a Hubble time.

From the observational point of view one can discriminate between the formation scenario as proposed in this project and the primordial one by measuring the dark matter content of dwarf objects.

\section{Acknowledgements}

MF acknowledges financial support through DFG-grant FE564/1-1.

\section{References}

Fellhauer M., Kroupa P., Baumgardt H., Bien R., Boily C.M., Spurzem R., Wassmer N., 2000, NewA, 5, 305

Fellhauer M., Baumgardt H., Kroupa P., Spurzem R., 2001, to appear in Cel. Mech. \& Dyn. Astron., astro-ph/0103052

Fellhauer M., Kroupa P., 2001, to appear in MNRAS, astro-ph/0110621

Gallagher S.C., Charlton J.C., Hunsberger S.D., Zaritsky D., Whitmore B.C., 2001, AJ, in press astro-ph/0104005

Hilker M., Infante L., Kissler-Patig M., Richtler T., 1999, A\&AS, 134, 75

Kroupa P., 1997, NewA, 2, 139

Mieske S., Hilker M., 2001, AG Abstract Series, 18, 215

Phillipps S., Drinkwater M.J., Gregg M.D., Jones J.B., 2001, to appear in ApJ, astro-ph/0106377

Whitmore B.C., Zhang Q., Leitherer C., Fall S.M., 1999, AJ, 118, 1551

Zhang Q., Fall S.M., 1999, ApJL, 527, 81L 